Article

\title{
Sampling Associated with a Unitary Representation of a Semi-Direct Product of Groups: A Filter Bank Approach
}

\author{
Antonio G. García ${ }^{1, *(0)}$, Miguel Angel Hernández-Medina ${ }^{2}$ [D and Gerardo Pérez-Villalón ${ }^{3}$ \\ 1 Departamento de Matemáticas, Universidad Carlos III de Madrid, Avda. de la Universidad 30, \\ 28911 Leganés-Madrid, Spain \\ 2 Information Processing and Telecommunications Center and Departamento de Matemática Aplicada a las \\ Tecnologías de la Información y las Comunicaciones, Universidad Politécnica de Madrid, Avda. \\ Complutense 30, 28040 Madrid, Spain; miguelangel.hernandez.medina@upm.es \\ 3 Departamento de Matemática Aplicada a las Tecnologías de la Información y las Comunicaciones, \\ Universidad Politécnica de Madrid, Nicola Tesla s/n, 28031 Madrid, Spain; gperez@euitt.upm.es \\ * Correspondence: agarcia@math.uc3m.es
}

Received: 18 March 2019; Accepted: 9 April 2019; Published: 12 April 2019

\begin{abstract}
An abstract sampling theory associated with a unitary representation of a countable discrete non abelian group $G$, which is a semi-direct product of groups, on a separable Hilbert space is studied. A suitable expression of the data samples, the use of a filter bank formalism and the corresponding frame analysis allow for fixing the mathematical problem to be solved: the search of appropriate dual frames for $\ell^{2}(G)$. An example involving crystallographic groups illustrates the obtained results by using either average or pointwise samples.
\end{abstract}

Keywords: semi-direct product of groups; unitary representation of a group; LCA groups; dual frames; sampling expansions

\section{Statement of the Problem}

In this paper, an abstract sampling theory associated with non abelian groups is derived for the specific case of a unitary representation of a semi-direct product of groups on a separable Hilbert space. Semi-direct product of groups provide important examples of non abelian groups such as dihedral groups, infinite dihedral group, Euclidean motion groups or crystallographic groups. Concretely, let $(n, h) \mapsto U(n, h)$ be a unitary representation on a separable Hilbert space $\mathcal{H}$ of a semi-direct product $G=N \rtimes_{\phi} H$, where $N$ is a countable discrete LCA (locally compact abelian) group, $H$ is a finite group, and $\phi$ denotes the action of the group $H$ on the group $N$ (see Section 2 infra for the details); for a fixed $a \in \mathcal{H}$ we consider the $U$-invariant subspace in $\mathcal{H}$

$$
\mathcal{A}_{a}=\left\{\sum_{(n, h) \in G} \alpha(n, h) U(n, h) a:\{\alpha(n, h)\}_{(n, h) \in G} \in \ell^{2}(G)\right\},
$$

where we assume that $\{U(n, h) a\}$ is a Riesz sequence for $\mathcal{H}$, i.e., a Riesz basis for $\mathcal{A}_{a}$ (see Ref. [1] for a necessary and sufficient condition). Given $K$ elements $b_{k}$ in $\mathcal{H}$, which do not belong necessarily to $\mathcal{A}_{a}$, the main goal in this paper is the stable recovery of any $x \in \mathcal{A}_{a}$ from the given data (generalized samples)

$$
\mathcal{L}_{k} x(n):=\left\langle x, U\left(n, 1_{H}\right) b_{k}\right\rangle_{\mathcal{H}}, \quad n \in N \text { and } k=1,2, \ldots, K,
$$


where $1_{H}$ denotes the identity element in $H$. These samples are nothing but a generalization of average sampling in shift-invariant subspaces of $L^{2}\left(\mathbb{R}^{d}\right)$; see, among others, Refs. [2-9]. The case where $G$ is a discrete LCA group and the samples are taken at a uniform lattice of $G$ has been solved in Ref. [10]; this work relies on the use of the Fourier analysis in the LCA group $G$ (see also Ref. [11]). In the case involved here, a classical Fourier analysis is not available and, consequently, we need to overcome this drawback.

Having in mind the filter bank formalism in discrete LCA groups (see, for instance, Refs. [12-14]), the given data $\left\{\mathcal{L}_{k} x(n)\right\}_{n \in N ; k=1,2, \ldots, K}$ can be expressed as the output of a suitable $K$-channel analysis filter bank corresponding to the input $\alpha=\{\alpha(n, h)\}_{(n, h) \in G}$ in $\ell^{2}(G)$. As a consequence, the problem consists of finding a synthesis part of the former filter bank allowing perfect reconstruction; in addition, only Fourier analysis on the LCA group $N$ is needed. Then, roughly speaking, substituting the output of the synthesis part in $x=\sum_{(n, h) \in G} \alpha(n, h) U(n, h) a$, we will obtain the corresponding sampling formula in $\mathcal{A}_{a}$.

This said, as it could be expected, the problem can be mathematically formulated as the search of dual frames for $\ell^{2}(G)$ having the form

$$
\left\{T_{n} \mathrm{~h}_{k}\right\}_{n \in N ; k=1,2, \ldots, K} \text { and }\left\{T_{n} \mathrm{~g}_{k}\right\}_{n \in N ; k=1,2, \ldots, K} .
$$

Here, $\mathrm{h}_{k}, \mathrm{~g}_{k} \in \ell^{2}(G), \mathrm{T}_{n} \mathrm{~h}_{k}(m, h)=\mathrm{h}_{k}(m-n, h)$ and $T_{n} \mathrm{~g}_{k}(m, h)=\mathrm{g}_{k}(m-n, h),(m, h) \in G$, where $n \in N$ and $k=1,2, \ldots, K$. In addition, for any $x \in \mathcal{A}_{a}$, we have the expression for its samples

$$
\mathcal{L}_{k} x(n)=\left\langle\boldsymbol{\alpha}, T_{n} \mathrm{~h}_{k}\right\rangle_{\ell^{2}(G)}, \quad n \in N \text { and } k=1,2, \ldots, K .
$$

Needless to say, frame theory plays a central role in what follows; the necessary background on Riesz bases or frame theory in a separable Hilbert space can be found, for instance, in Ref. [15]. Finally, sampling formulas in $\mathcal{A}_{a}$ having the form

$$
x=\sum_{k=1}^{K} \sum_{n \in N} \mathcal{L}_{k} x(n) U\left(n, 1_{H}\right) c_{k} \quad \text { in } \mathcal{H},
$$

for some $c_{k} \in \mathcal{A}_{a}, k=1,2, \ldots, K$, will come out by using, for $\mathrm{g} \in \ell^{2}(G)$ and $n \in N$, the shifting property $\mathcal{T}_{U, a}\left(T_{n} \mathrm{~g}\right)=U\left(n, 1_{H}\right)\left(\mathcal{T}_{U, a} \mathrm{~g}\right)$ that satisfies the natural isomorphism $\mathcal{T}_{U, a}: \ell^{2}(G) \rightarrow \mathcal{A}_{a}$ which maps the usual orthonormal basis $\left\{\delta_{(n, h)}\right\}_{(n, h) \in G}$ for $\ell^{2}(G)$ onto the Riesz basis $\{U(n, h) a\}_{(n, h) \in G}$ for $\mathcal{A}_{a}$. All these steps will be carried out throughout the remaining sections. For the sake of completeness, Section 2 includes some basic preliminaries on semi-direct product of groups and Fourier analysis on LCA groups. The paper ends with an illustrative example involving the quasi regular representation of a crystallographic group on $L^{2}\left(\mathbb{R}^{d}\right)$; sampling formulas involving average or pointwise samples are obtained for the corresponding $U$-invariant subspaces in $L^{2}\left(\mathbb{R}^{d}\right)$.

\section{Some Mathematical Preliminaries}

In this section, we introduce the basic tools in semi-direct product of groups and in harmonic analysis in a discrete LCA group that will be used in the sequel.

\subsection{Preliminaries on Semi-Direct Product of Groups}

Given groups $(N, \cdot)$ and $(H, \cdot)$, and a homomorphism $\phi: H \rightarrow A u t(N)$, their semi-direct product $G:=N \rtimes_{\phi} H$ is defined as follows: The underlying set of $G$ is the set of pairs $(n, h)$ with $n \in N$ and $h \in H$, along with the multiplication rule

$$
\left(n_{1}, h_{1}\right) \cdot\left(n_{2}, h_{2}\right):=\left(n_{1} \phi_{h_{1}}\left(n_{2}\right), h_{1} h_{2}\right), \quad\left(n_{1}, h_{1}\right),\left(n_{2}, h_{2}\right) \in G,
$$


where we denote $\phi(h):=\phi_{h}$; usually, the homomorphism $\phi$ is referred to as the action of the group $H$ on the group $N$. Thus, we obtain a new group with identity element $\left(1_{N}, 1_{H}\right)$, and inverse $(n, h)^{-1}=\left(\phi_{h^{-1}}\left(n^{-1}\right), h^{-1}\right)$.

In addition, we have the isomorphisms $N \simeq N \times\left\{1_{H}\right\}$ and $H \simeq\left\{1_{N}\right\} \times H$. Unless $\phi_{h}$ equals the identity for all $h \in H$, the group $G=N \rtimes_{\phi} H$ is not abelian, even for abelian $N$ and $H$ groups. The subgroup $N$ is a normal subgroup in $G$. Some examples of semi-direct product of groups:

1. The dihedral group $D_{2 N}$ is the group of symmetries of a regular $N$-sided polygon; it is the semi-direct product $D_{2 N}=\mathbb{Z}_{N} \rtimes_{\phi} \mathbb{Z}_{2}$ where $\phi_{\overline{0}} \equiv I d_{\mathbb{Z}_{N}}$ and $\phi_{\overline{1}}(\bar{n})=-\bar{n}$ for each $\bar{n} \in \mathbb{Z}_{N}$. The infinite dihedral group $D_{\infty}$ defined as $\mathbb{Z} \rtimes_{\phi} \mathbb{Z}_{2}$ for the similar homomorphism $\phi$ is the group of isometries of $\mathbb{Z}$.

2. The Euclidean motion group $E(d)$ is the semi-direct product $\mathbb{R}^{d} \rtimes_{\phi} O(d)$, where $O(d)$ is the orthogonal group of order $d$ and $\phi_{A}(x)=A x$ for $A \in O(d)$ and $x \in \mathbb{R}^{d}$. It contains as a subgroup any crystallographic group $M \mathbb{Z}^{d} \rtimes_{\phi} \Gamma$, where $M \mathbb{Z}^{d}$ denotes a full rank lattice of $\mathbb{R}^{d}$ and $\Gamma$ is any finite subgroup of $O(d)$ such that $\phi_{\gamma}\left(M \mathbb{Z}^{d}\right)=M \mathbb{Z}^{d}$ for each $\gamma \in \Gamma$.

3. The orthogonal group $O(d)$ of all orthogonal real $d \times d$ matrices is isomorphic to the semi-direct product $S O(d) \rtimes_{\phi} C_{2}$, where $S O(d)$ consists of all orthogonal matrices with determinant 1 and $C_{2}=\{I, R\}$ a cyclic group of order $2 ; \phi$ is the homomorphism given by $\phi_{I}(A)=A$ and $\phi_{R}(A)=$ $R A R^{-1}$ for $A \in S O(d)$.

Suppose that $N$ is an LCA group with Haar measure $\mu_{N}$ and $H$ is a locally compact group with Haar measure $\mu_{H}$. Then, the semi-direct product $G=N \rtimes_{\phi} H$ endowed with the product topology becomes also a topological group. For the left Haar measure on $G$, see Ref. [1].

\subsection{Some Preliminaries on Harmonic Analysis on Discrete LCA Groups}

The results about harmonic analysis on locally compact abelian (LCA) groups are borrowed from Ref. [16]. Notice that, in particular, a countable discrete abelian group is a second countable Hausdorff LCA group.

For a countable discrete group $(N, \cdot)$, not necessarily abelian, the convolution of $x, y: N \rightarrow \mathbb{C}$ is formally defined as $(x * y)(m):=\sum_{n \in N} x(n) y\left(n^{-1} m\right), m \in N$. If, in addition, the group is abelian, therefore denoted by $(N,+)$, the convolution reads as

$$
(x * y)(m):=\sum_{n \in N} x(n) y(m-n), \quad m \in N .
$$

Let $\mathbb{T}=\{z \in \mathbb{C}:|z|=1\}$ be the unidimensional torus. We said that $\xi: N \mapsto \mathbb{T}$ is a character of $N$ if $\xi(n+m)=\xi(n) \xi(m)$ for all $n, m \in N$. We denote $\xi(n)=\langle n, \xi\rangle$. Defining $(\xi+\gamma)(n)=\xi(n) \gamma(n)$, the set of characters $\widehat{N}$ with the operation + is a group, called the dual group of $N$; since $N$ is discrete $\widehat{N}$ is compact ([16], Prop. 4.4). For $x \in \ell^{1}(N)$, we define its Fourier transform as

$$
X(\xi)=\widehat{x}(\xi):=\sum_{n \in N} x(n) \overline{\langle n, \xi\rangle}=\sum_{n \in N} x(n)\langle-n, \xi\rangle, \quad \xi \in \widehat{N} .
$$

It is known ([16], Theorem 4.5) that $\widehat{\mathbb{Z}} \cong \mathbb{T}$, with $\langle n, z\rangle=z^{n}$, and $\widehat{\mathbb{Z}}_{s} \cong \mathbb{Z}_{s}:=\mathbb{Z} / s \mathbb{Z}$, with $\langle n, m\rangle=W_{s}^{n m}$, where $W_{s}=e^{2 \pi i / s}$.

There exists a unique measure, the Haar measure $\mu$ on $\widehat{N}$ satisfying $\mu(\xi+E)=\mu(E)$, for every Borel set $E \subset \widehat{N}\left([16]\right.$, Section 2.2), and $\mu(\widehat{N})=1$. We denote $\int_{\widehat{N}} X(\xi) d \xi=\int_{\widehat{N}} X(\xi) d \mu(\xi)$. If $N=\mathbb{Z}$,

$$
\int_{\widehat{N}} X(\xi) d \xi=\int_{\mathbb{T}} X(z) d z=\frac{1}{2 \pi} \int_{0}^{2 \pi} X\left(e^{i w}\right) d w
$$

and, if $N=\mathbb{Z}_{s}$,

$$
\int_{\widehat{N}} X(\xi) d \xi=\int_{\mathbb{Z}_{s}} X(n) d n=\frac{1}{s} \sum_{n \in \mathbb{Z}_{s}} X(n)
$$


If $N_{1}, N_{2}, \ldots N_{d}$ are abelian discrete groups, then the dual group of the product group is $\left(N_{1} \times\right.$ $\left.N_{2} \times \ldots \times N_{d}\right)^{\wedge} \cong \widehat{N}_{1} \times \widehat{N}_{2} \times \ldots \times \widehat{N}_{d}$ (see ([16], Prop. 4.6)) with

$$
\left\langle\left(n_{1}, n_{2}, \ldots, n_{d}\right),\left(\xi_{1}, \xi_{2} \ldots, \xi_{d}\right)\right\rangle=\left\langle n_{1}, \xi_{1}\right\rangle\left\langle n_{2}, \xi_{2}\right\rangle \cdots\left\langle n_{d}, \xi_{d}\right\rangle .
$$

The Fourier transform on $\ell^{1}(N) \cap \ell^{2}(N)$ is an isometry on a dense subspace of $L^{2}(\widehat{N})$; Plancherel theorem extends it in a unique manner to a unitary operator of $\ell^{2}(N)$ onto $L^{2}(\widehat{N})$ ([16], p. 99). The following lemma, giving a relationship between Fourier transform and convolution, will be used later (see Ref. [17]):

Lemma 1. Assume that $a, b \in \ell^{2}(N)$ and $\widehat{a}(\xi) \widehat{b}(\xi) \in L^{2}(\widehat{N})$. Then, the convolution $a * b$ belongs to $\ell^{2}(N)$ and $\widehat{a * b}(\xi)=\widehat{a}(\xi) \widehat{b}(\xi)$, a.e. $\xi \in \widehat{N}$.

\section{Filter Bank Formalism on Semi-Direct Product of Groups}

In what follows, we will assume that $G=N \rtimes_{\phi} H$ where $(N,+)$ is a countable discrete abelian group and $(H, \cdot)$ is a finite group. Having in mind the operational calculus $(n, h) \cdot(m, l)=\left(n+\phi_{h}(m), h l\right)$, $(n, h)^{-1}=\left(\phi_{h^{-1}}(-n), h^{-1}\right)$ and $(n, h)^{-1} \cdot(m, l)=\left(\phi_{h^{-1}}(m-n), h^{-1} l\right)$, the convolution $\boldsymbol{\alpha} * \mathrm{~h}$ of $\boldsymbol{\alpha}, \mathrm{h} \in \ell^{2}(G)$ can be expressed as

$$
\begin{aligned}
(\boldsymbol{\alpha} * \mathrm{~h})(m, l) & =\sum_{(n, h) \in G} \alpha(n, h) \mathrm{h}\left[(n, h)^{-1} \cdot(m, l)\right] \\
& =\sum_{(n, h) \in G} \alpha(n, h) \mathrm{h}\left(\phi_{h^{-1}}(m-n), h^{-1} l\right), \quad(m, l) \in G .
\end{aligned}
$$

For a function $\boldsymbol{\alpha}: G \rightarrow \mathbb{C}$, its $H$-decimation $\downarrow_{H} \boldsymbol{\alpha}: N \rightarrow \mathbb{C}$ is defined as $\left(\downarrow_{H} \boldsymbol{\alpha}\right)(n):=\alpha\left(n, 1_{H}\right)$ for any $n \in N$. Thus, we have

$$
\begin{aligned}
\downarrow_{H}(\boldsymbol{\alpha} * \mathrm{~h})(m) & =(\boldsymbol{\alpha} * \mathrm{~h})\left(m, 1_{H}\right)=\sum_{(n, h) \in G} \alpha(n, h) \mathrm{h}\left(\phi_{h^{-1}}(m-n), h^{-1}\right) \\
& =\sum_{(n, h) \in G} \alpha(n, h) \mathrm{h}\left[(n-m, h)^{-1}\right], \quad m \in N .
\end{aligned}
$$

Defining the polyphase components of $\boldsymbol{\alpha}$ and $\mathrm{h}$ as $\boldsymbol{\alpha}_{h}(n):=\alpha(n, h)$ and $\mathrm{h}_{h}(n):=\mathrm{h}\left[(-n, h)^{-1}\right]$ respectively, we write

$$
\downarrow_{H}(\boldsymbol{\alpha} * \mathrm{~h})(m)=\sum_{h \in H} \sum_{n \in N} \boldsymbol{\alpha}_{h}(n) \mathrm{h}_{h}(m-n)=\sum_{h \in H}\left(\boldsymbol{\alpha}_{h} *_{N} \mathrm{~h}_{h}\right)(m), \quad m \in N .
$$

For a function $c: N \rightarrow \mathbb{C}$, its $H$-expander $\uparrow_{H} c: G \rightarrow \mathbb{C}$ is defined as

$$
\left(\uparrow_{H} c\right)(n, h)= \begin{cases}c(n) & \text { if } h=1_{H}, \\ 0 & \text { if } h \neq 1_{H} .\end{cases}
$$

In case $\uparrow_{H} c$ and g belong to $\ell^{2}(G)$, we have

$$
\begin{aligned}
\left(\uparrow_{H} \mathcal{c} * \mathrm{~g}\right)(m, l) & =\sum_{(n, h) \in G}\left(\uparrow_{H} c\right)(n, h) \mathrm{g}\left[(n, h)^{-1} \cdot(m, l)\right] \\
& =\sum_{(n, h) \in G}\left(\uparrow_{H} c\right)(n, h) \mathrm{g}\left(\phi_{h^{-1}}(m-n), h^{-1} l\right) \\
& =\sum_{n \in N} c(n) \mathrm{g}(m-n, l)=\left(c *_{N} \mathrm{~g}_{l}\right)(m), \quad m \in N, l \in H,
\end{aligned}
$$

where $\mathrm{g}_{l}(n):=\mathrm{g}(n, l)$ is the polyphase component of $\mathrm{g}$. 
From now on, we will refer to a K-channel filter bank with analysis filters $\mathrm{h}_{k}$ and synthesis filters $\mathrm{g}_{k}$, $k=1,2, \ldots, K$ as the one given by (see Figure 1 )

$$
\mathbf{c}_{k}:=\downarrow_{H}\left(\boldsymbol{\alpha} * \mathrm{~h}_{k}\right), \quad k=1,2, \ldots, K \text {, and } \boldsymbol{\beta}=\sum_{k=1}^{K}\left(\uparrow_{H} c_{k}\right) * \mathrm{~g}_{k} \text {, }
$$

where $\alpha$ and $\beta$ denote, respectively, the input and the output of the filter bank. In polyphase notation,

$$
\begin{aligned}
& \mathbf{c}_{k}(m)=\sum_{h \in H}\left(\boldsymbol{\alpha}_{h} *_{N} \mathrm{~h}_{k, h}\right)(m), \quad m \in N, \quad k=1,2, \ldots, K, \\
& \boldsymbol{\beta}_{l}(m)=\sum_{k=1}^{K}\left(\mathbf{c}_{k} *_{N} \mathrm{~g}_{l, k}\right)(m), \quad m \in N, l \in H
\end{aligned}
$$

where $\alpha_{h}(n):=\alpha(n, h), \beta_{l}(n):=\beta(n, l), \mathrm{h}_{k, h}(n):=\mathrm{h}_{k}\left[(-n, h)^{-1}\right]$ and $\mathrm{g}_{l, k}(n):=\mathrm{g}_{k}(n, l)$ are the polyphase components of $\boldsymbol{\alpha}, \boldsymbol{\beta}, \mathrm{h}_{k}$ and $\mathrm{g}_{k}, k=1,2, \ldots, K$, respectively. We also assume that $\mathrm{h}_{k}, \mathrm{~g}_{k} \in \ell^{2}(G)$ with $\widehat{h}_{k, h}, \widehat{\mathrm{g}}_{h, k} \in L^{\infty}(\widehat{N})$ for $k=1,2, \ldots, K$ and $h \in H$; from Lemma 1, the filter bank (3) is well defined in $\ell^{2}(G)$.

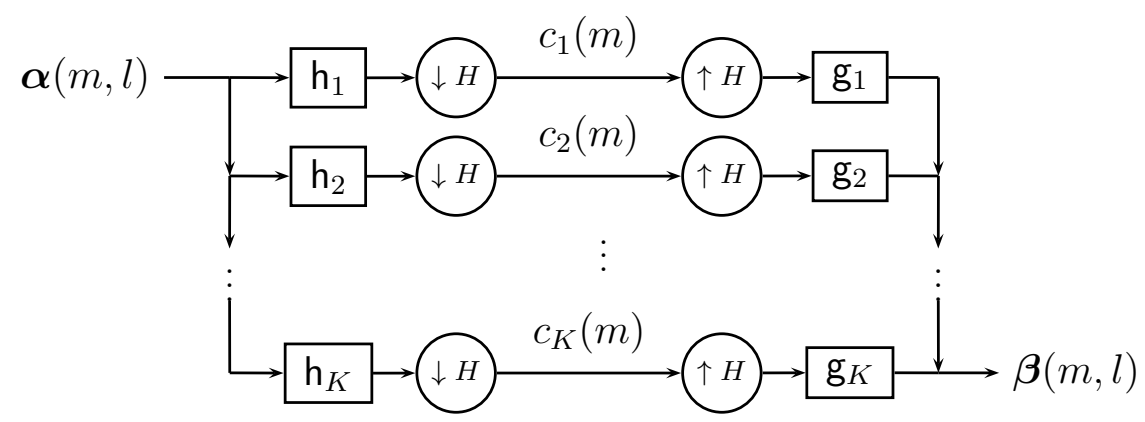

Figure 1. The K-channel filter bank scheme.

The above K-channel filter bank (3) is said to be a perfect reconstruction filter bank if and only if it satisfies $\alpha=\sum_{k=1}^{K}\left(\uparrow_{H} c_{k}\right) * g_{k}$ for each $\alpha \in \ell^{2}(G)$, or equivalently, $\alpha_{h}=\sum_{k=1}^{K}\left(c_{k} *_{N} g_{h, k}\right)$ for each $h \in H$.

Since $N$ is an LCA group where a Fourier transform is available, the polyphase expression (4) of the filter bank (3) allows us to carry out its polyphase analysis.

\section{Polyphase Analysis: Perfect Reconstruction Condition}

For notational ease, we denote $L:=|H|$, the order of the group $H$, and its elements as $H=\left\{h_{1}, h_{2}, \ldots, h_{L}\right\}$. Having in mind Lemma 1, the $N$-Fourier transform in $\mathbf{c}_{k}(m)=\sum_{h \in H}\left(\boldsymbol{\alpha}_{h} *_{N}\right.$ $\left.\mathrm{h}_{k, h}\right)(m)$ gives $\widehat{\mathbf{c}}_{k}(\gamma)=\sum_{h \in H} \widehat{\mathrm{h}}_{k, h}(\gamma) \widehat{\boldsymbol{\alpha}}_{h}(\gamma)$ a.e. $\gamma \in \widehat{N}$ for each $k=1,2, \ldots, K$. In matrix notation,

$$
\mathbf{C}(\gamma)=\mathbf{H}(\gamma) \mathbf{A}(\gamma) \quad \text { a.e. } \gamma \in \widehat{N}
$$

where $\mathbf{C}(\gamma)=\left(\widehat{\mathbf{c}}_{1}(\gamma), \widehat{\mathbf{c}}_{2}(\gamma), \ldots, \widehat{\mathbf{c}}_{K}(\gamma)\right)^{\top}, \mathbf{A}(\gamma)=\left(\widehat{\boldsymbol{\alpha}}_{h_{1}}(\gamma), \widehat{\boldsymbol{\alpha}}_{h_{2}}(\gamma), \ldots, \widehat{\boldsymbol{\alpha}}_{h_{L}}(\gamma)\right)^{\top}$, and $\mathbf{H}(\gamma)$ is the $K \times L$ matrix

$$
\mathbf{H}(\gamma)=\left(\begin{array}{cccc}
\widehat{\mathrm{h}}_{1, h_{1}}(\gamma) & \widehat{\mathrm{h}}_{1, h_{2}}(\gamma) & \cdots & \widehat{\mathrm{h}}_{1, h_{L}}(\gamma) \\
\widehat{\mathrm{h}}_{2, h_{1}}(\gamma) & \widehat{\mathrm{h}}_{2, h_{2}}(\gamma) & \cdots & \widehat{\mathrm{h}}_{2, h_{L}}(\gamma) \\
\cdots & \cdots & \cdots & \cdots \\
\widehat{\mathrm{h}}_{K, h_{1}}(\gamma) & \widehat{\mathrm{h}}_{K, h_{2}}(\gamma) & \cdots & \widehat{\mathrm{h}}_{K, h_{L}}(\gamma)
\end{array}\right)
$$

where $\widehat{\mathrm{h}}_{k, h_{i}} \in L^{2}(\widehat{N})$ is the Fourier transform of $\mathrm{h}_{k, h_{i}}(n):=\mathrm{h}_{k}\left[\left(-n, h_{i}\right)^{-1}\right] \in \ell^{2}(N)$. 
The same procedure for $\boldsymbol{\beta}_{l}(m)=\sum_{k=1}^{K}\left(\mathbf{c}_{k} *_{N} \mathbf{g}_{l, k}\right)(m)$ gives $\widehat{\boldsymbol{\beta}}_{l}(\gamma)=\sum_{k=1}^{K} \widehat{\mathbf{g}}_{l, k}(\gamma) \widehat{\mathbf{c}}_{k}(\gamma)$ a.e. $\gamma \in \widehat{N}$. In matrix notation,

$$
\mathbf{B}(\gamma)=\mathbf{G}(\gamma) \mathbf{C}(\gamma) \text { a.e. } \gamma \in \widehat{N}
$$

where $\mathbf{B}(\gamma)=\left(\widehat{\boldsymbol{\beta}}_{h_{1}}(\gamma), \widehat{\boldsymbol{\beta}}_{h_{2}}(\gamma), \ldots, \widehat{\boldsymbol{\beta}}_{h_{L}}(\gamma)\right)^{\top}, \mathbf{C}(\gamma)=\left(\widehat{\mathbf{c}}_{1}(\gamma), \widehat{\mathbf{c}}_{2}(\gamma), \ldots, \widehat{\mathbf{c}}_{K}(\gamma)\right)^{\top}$ and $\mathbf{G}(\gamma)$ is the $L \times K$ matrix

$$
\mathbf{G}(\gamma)=\left(\begin{array}{cccc}
\widehat{\mathrm{g}}_{h_{1}, 1}(\gamma) & \widehat{\mathrm{g}}_{h_{1,2}}(\gamma) & \ldots & \widehat{\mathrm{g}}_{h_{1}, K}(\gamma) \\
\widehat{\mathrm{g}}_{h_{2}, 1}(\gamma) & \widehat{\mathrm{g}}_{h_{2}, 2}(\gamma) & \ldots & \widehat{\mathrm{g}}_{h_{2}, K}(\gamma) \\
\ldots & \ldots & \ldots & \ldots \\
\widehat{\mathrm{g}}_{h_{L}, 1}(\gamma) & \widehat{\mathrm{g}}_{h_{L}, 2}(\gamma) & \ldots & \widehat{\mathrm{g}}_{h_{L}, K}(\gamma)
\end{array}\right),
$$

where $\widehat{\mathrm{g}}_{h_{i}, k} \in L^{2}(\widehat{N})$ is the Fourier transform of $\mathrm{g}_{h_{i}, k}(n):=\mathrm{g}_{k}\left(n, h_{i}\right) \in \ell^{2}(N)$.

Thus, in terms of the polyphase matrices $\mathbf{G}(\gamma)$ and $\mathbf{H}(\gamma)$, the filter bank (3) can be expressed as

$$
\mathbf{B}(\gamma)=\mathbf{G}(\gamma) \mathbf{H}(\gamma) \mathbf{A}(\gamma) \text { a.e. } \gamma \in \widehat{N} .
$$

As a consequence of Equation (7), we have:

Theorem 1. The K-channel filter bank given in Equation (3), where $\mathrm{h}_{k}, \mathrm{~g}_{k}$ belong to $\ell^{2}(G)$ and $\widehat{\mathrm{h}}_{k, h_{i}}, \widehat{\mathrm{g}}_{h_{h}, k}$ belong to $L^{\infty}(\widehat{N})$ for $k=1,2, \ldots, K$ and $i=1,2, \ldots, L$, satisfies the perfect reconstruction property if and only if $\mathbf{G}(\gamma) \mathbf{H}(\gamma)=\mathbf{I}_{L}$ a.e. $\gamma \in \widehat{N}$, where $\mathbf{I}_{L}$ denotes the identity matrix of order $L$.

Proof. First of all, note that the mapping $\alpha \in \ell^{2}(G) \mapsto \mathbf{A} \in L_{L}^{2}(\widehat{N})$ is a unitary operator. Indeed, for each $\alpha, \beta \in \ell^{2}(G)$, we have the isometry property

$$
\begin{aligned}
\langle\boldsymbol{\alpha}, \boldsymbol{\beta}\rangle_{\ell^{2}(G)} & =\sum_{(m, h) \in G} \alpha(m, h) \overline{\beta(m, h)}=\sum_{h \in H}\left\langle\boldsymbol{\alpha}_{h}, \boldsymbol{\beta}_{h}\right\rangle_{\ell^{2}(N)} \\
& =\sum_{h \in H}\left\langle\widehat{\boldsymbol{\alpha}}_{h}, \widehat{\boldsymbol{\beta}}_{h}\right\rangle_{L^{2}(\widehat{N})}=\langle\mathbf{A}, \mathbf{B}\rangle_{L_{L}^{2}(\widehat{N})} .
\end{aligned}
$$

It is also surjective since the $N$-Fourier transform is a surjective isometry between $\ell^{2}(N)$ and $L^{2}(\widehat{N})$. Having in mind this property, Equation (7) tells us that the filter bank satisfies the perfect reconstruction property if and only if $\mathbf{G}(\gamma) \mathbf{H}(\gamma)=\mathbf{I}_{L}$ a.e. $\gamma \in \widehat{N}$.

Notice that, in the perfect reconstruction setting, the number of channels $K$ must be necessarily bigger or equal that the order $L$ of the group $H$, i.e., $K \geq L$.

\section{Frame Analysis}

For $m \in N$, the translation operator $T_{m}: \ell^{2}(G) \rightarrow \ell^{2}(G)$ is defined as

$$
T_{m} \alpha(n, h):=\alpha\left(\left(m, 1_{H}\right)^{-1} \cdot(n, h)\right)=\alpha(n-m, h),(n, h) \in G .
$$

The involution operator $\alpha \in \ell^{2}(G) \mapsto \widetilde{\boldsymbol{\alpha}} \in \ell^{2}(G)$ is defined as $\widetilde{\alpha}(n, h):=\overline{\alpha\left((n, h)^{-1}\right)},(n, h) \in G$. As expected, the classical relationship between convolution and translation operators holds. Thus, for the K-channel filter bank (3), we have (see (2)):

$$
\mathbf{c}_{k}(m)=\downarrow_{H}\left(\boldsymbol{\alpha} * \mathrm{~h}_{k}\right)(m)=\left\langle\boldsymbol{\alpha}, T_{m} \widetilde{\mathrm{h}}_{k}\right\rangle_{\ell^{2}(G)}, \quad m \in N, k=1,2, \ldots, K .
$$

In addition,

$$
\left(\uparrow_{H} \mathbf{c}_{k} * \mathrm{~g}_{k}\right)(m, h)=\sum_{n \in N} \mathbf{c}_{k}(n) \mathrm{g}_{k}(m-n, h)=\sum_{n \in N}\left\langle\boldsymbol{\alpha}, T_{n} \widetilde{\mathrm{h}}_{k}\right\rangle_{\ell^{2}(G)} T_{n} \mathrm{~g}_{k}(m, h) .
$$


In the perfect reconstruction setting, for any $\alpha \in \ell^{2}(G)$, we have

$$
\boldsymbol{\alpha}=\sum_{k=1}^{K} \sum_{n \in N}\left\langle\boldsymbol{\alpha}, T_{n} \widetilde{\mathrm{h}}_{k}\right\rangle_{\ell^{2}(G)} T_{n} \mathrm{~g}_{k} \quad \text { in } \ell^{2}(G) .
$$

Given $K$ sequences $\mathrm{f}_{k} \in \ell^{2}(G), k=1,2, \ldots, K$, our main tasks now are: $(i)$ to characterize the sequence $\left\{T_{n} \mathrm{f}_{k}\right\}_{n \in N ; k=1,2, \ldots, K}$ as a frame for $\ell^{2}(G)$, and (ii) to find its dual frames having the form $\left\{T_{n} \mathrm{~g}_{k}\right\}_{n \in N ; k=1,2, \ldots, K^{\prime}}$

To the first end, we consider a $K$-channel analysis filter bank with analysis filters $h_{k}:=\widetilde{f}_{k}$, i.e., the involution of $\mathrm{f}_{k}, k=1,2, \ldots, K$; let $\mathbf{H}(\gamma)$ be its associated $K \times L$ polyphase matrix (5). First, we check that Equation (5) is:

$$
\mathbf{H}(\gamma)=\left(\overline{\widehat{f}_{k, h_{i}}(\gamma)}\right)_{\substack{k=1,2, \ldots, K \\ i=1,2, \ldots, L}}
$$

where $\widehat{f}_{k, h_{i}}(\gamma)$ denotes the Fourier transform in $L^{2}(\widehat{N})$ of $\mathrm{f}_{k, h_{i}}(n)=\mathrm{f}_{k}\left(n, h_{i}\right)$ in $\ell^{2}(N)$. Indeed, for $k=1,2, \ldots, K$ and $i=1,2, \ldots, L$, having in mind that $\mathrm{h}_{k, h_{i}}(n)=\mathrm{h}_{k}\left[\left(-n, h_{i}\right)^{-1}\right]$ for analysis filters, we have

$$
\begin{aligned}
\widehat{\mathrm{h}}_{k, h_{i}}(\gamma) & =\sum_{n \in N} \mathrm{~h}_{k, h_{i}}(n)\langle-n, \gamma\rangle=\sum_{n \in N} \mathrm{~h}_{k}\left[\left(-n, h_{i}\right)^{-1}\right]\langle-n, \gamma\rangle=\sum_{n \in N} \tilde{f}_{k}\left[\left(-n, h_{i}\right)^{-1}\right]\langle-n, \gamma\rangle \\
& =\sum_{n \in N} \overline{\mathrm{f}_{k}\left(-n, h_{i}\right)}\langle-n, \gamma\rangle=\overline{\sum_{n \in N} \mathrm{f}_{k}\left(n, h_{i}\right)\langle-n, \gamma\rangle}=\overline{\mathfrak{f}_{k, h_{i}}(\gamma)}, \quad \gamma \in \widehat{N} .
\end{aligned}
$$

Next, we consider its associated constants

$$
A_{\mathbf{H}}:=\underset{\gamma \in \widehat{N}}{\operatorname{essinf}} \lambda_{\min }\left[\mathbf{H}^{*}(\gamma) \mathbf{H}(\gamma)\right] \quad \text { and } \quad B_{\mathbf{H}}:=\underset{\gamma \in \widehat{N}}{\operatorname{ess} \sup } \lambda_{\max }\left[\mathbf{H}^{*}(\gamma) \mathbf{H}(\gamma)\right]
$$

Theorem 2. For $\mathrm{f}_{k}$ in $\ell^{2}(G), k=1,2, \ldots, K$, consider the associated matrix $\mathbf{H}(\gamma)$ given in Equation (10). Then,

1. The sequence $\left\{T_{n} f_{k}\right\}_{n \in N ; k=1,2, \ldots, K}$ is a Bessel sequence for $\ell^{2}(G)$ if and only if $B_{\mathbf{H}}<\infty$.

2. The sequence $\left\{T_{n} \mathrm{f}_{k}\right\}_{n \in N ; k=1,2, \ldots, K}$ is a frame for $\ell^{2}(G)$ if and only if the inequalities $0<A_{\mathbf{H}} \leq B_{\mathbf{H}}<\infty$ hold.

Proof. Using Plancherel theorem ([16], Theorem 4.25), for each $\alpha \in \ell^{2}(G)$, we get

$$
\begin{aligned}
\left\langle\boldsymbol{\alpha}, T_{n} \mathrm{f}_{k}\right\rangle_{\ell^{2}(G)} & =\sum_{h \in H}\left\langle\boldsymbol{\alpha}_{h}, \mathrm{f}_{k, h}(\cdot-n)\right\rangle_{\ell^{2}(N)}=\sum_{h \in H} \int_{\widehat{N}} \widehat{\boldsymbol{\alpha}}_{h}(\gamma) \overline{\widehat{\mathrm{f}}_{k, h}(\gamma)\langle-n, \gamma\rangle} d \gamma \\
& =\int_{\widehat{N}} \sum_{h \in H} \widehat{\boldsymbol{\alpha}}_{h}(\gamma) \overline{\widehat{\mathrm{f}}_{k, h}(\gamma)} \overline{\langle-n, \gamma\rangle} d \gamma=\int_{\widehat{N}} \mathbf{H}_{k}(\gamma) \mathbf{A}(\gamma) \overline{\langle-n, \gamma\rangle} d \gamma,
\end{aligned}
$$

where $\mathbf{A}(\gamma)=\left(\widehat{\boldsymbol{\alpha}}_{h_{1}}(\gamma), \widehat{\boldsymbol{\alpha}}_{h_{2}}(\gamma), \ldots, \widehat{\boldsymbol{\alpha}}_{h_{L}}(\gamma)\right)^{\top}$ and $\mathbf{H}_{k}(\gamma)$ denotes the $k$-th row of $\mathbf{H}(\gamma)$.

Since $\{\langle-n, \gamma\rangle\}_{n \in N}$ is an orthonormal basis for $L^{2}(\widehat{N})$, in case that $\mathbf{H}(\gamma) \mathbf{A}(\gamma) \in L_{K}^{2}(\widehat{N})$, we have

$$
\sum_{k=1}^{K} \sum_{n \in N}\left|\left\langle\boldsymbol{\alpha}, T_{n} \mathrm{f}_{k}\right\rangle\right|^{2}=\sum_{k=1}^{K} \int_{\widehat{N}}\left|\mathbf{H}_{k}(\gamma) \mathbf{A}(\gamma)\right|^{2} d \gamma=\int_{\widehat{N}}\|\mathbf{H}(\gamma) \mathbf{A}(\gamma)\|^{2} d \gamma
$$

If $B_{\mathbf{H}}<\infty$, having in mind that $\|\boldsymbol{\alpha}\|_{\ell^{2}(G)}^{2}=\|\mathbf{A}\|_{L_{L}^{2}(\widehat{N})}^{2}=\int_{\widehat{N}}\|\mathbf{A}(\gamma)\|^{2} d \gamma$, the above equality and the Rayleigh-Ritz theorem ([18], Theorem 4.2.2) prove that $\left\{T_{n} \mathrm{f}_{k}\right\}_{n \in N ; k=1,2, \ldots, K}$ is a Bessel sequence for $\ell^{2}(G)$ with Bessel bound less or equal than $B_{\mathbf{H}}$. 
On the other hand, if $K<B_{\mathbf{H}}$, then there exists a set $\Omega \subset \widehat{N}$ having a strictly positive measure such that $\lambda_{\max }\left[\mathbf{H}^{*}(\gamma) \mathbf{H}(\gamma)\right]>K$ for $\gamma \in \Omega$. Consider $\boldsymbol{\alpha}$ such that its associated $\mathbf{A}(\gamma)$ is 0 if $\gamma \notin \Omega$, and $\mathbf{A}(\gamma)$ is a unitary eigenvector corresponding to the largest eigenvalue of $\mathbf{H}^{*}(\gamma) \mathbf{H}(\gamma)$ if $\gamma \in \Omega$. Thus, we have that

$$
\sum_{k=1}^{K} \sum_{n \in N}\left|\left\langle\boldsymbol{\alpha}, T_{n} \mathrm{f}_{k}\right\rangle\right|^{2}=\int_{\widehat{N}}\|\mathbf{H}(\gamma) \mathbf{A}(\gamma)\|^{2} d \gamma>K \int_{\widehat{N}}\|\mathbf{A}(\gamma)\|^{2} d \gamma=K\|\boldsymbol{\alpha}\|_{\ell^{2}(G)}^{2}
$$

As a consequence, if $B_{\mathbf{H}}=\infty$, the sequence is not Bessel, and, if $B_{\mathbf{H}}<\infty$, the optimal bound is precisely $B_{\mathbf{H}}$.

Similarly, by using inequality $\|\mathbf{H}(\gamma) \mathbf{A}(\gamma)\|^{2} \geq \lambda_{\min }\left[\mathbf{H}^{*}(\gamma) \mathbf{H}(\gamma)\right]\|\mathbf{A}(\gamma)\|^{2}$, and that equality holds whenever $\mathbf{A}(\gamma)$ is a unitary eigenvector corresponding to the smallest eigenvalue of $\mathbf{H}^{*}(\gamma) \mathbf{H}(\gamma)$; one proves the other inequality in part 2 .

Corollary 1. The sequence $\left\{T_{n} \mathrm{f}_{k}\right\}_{n \in N ; k=1,2, \ldots, K}$ is a Bessel sequence for $\ell^{2}(G)$ if and only if for each $k=1,2, \ldots, K$ and $i=1,2, \ldots, L$ the function $\widehat{\mathrm{f}}_{k, h_{i}}$ belongs to $L^{\infty}(\widehat{N})$.

Proof. It is a direct consequence of the equivalence between the spectral and Frobenius norms for matrices [18].

It is worth mentioning that $f_{k}$ in $\ell^{1}(G), k=1,2, \ldots, K$, implies that the sequence $\left\{T_{n} \mathrm{f}_{k}\right\}_{n \in N ; k=1,2, \ldots, K}$ is always a Bessel sequence for $\ell^{2}(G)$ since each function $\widehat{\mathrm{f}}_{k, h_{i}}$ is continuous and $\widehat{N}$ is compact. In this case, the frame condition for $\left\{T_{n} \mathrm{f}_{k}\right\}_{n \in N ; k=1,2, \ldots, K}$ reduces to rank $\mathbf{H}(\gamma)=L$ for all $\gamma \in \widehat{N}$ or, equivalently,

$$
\min _{\gamma \in \widehat{N}}\left(\operatorname{det}\left[\mathbf{H}^{*}(\gamma) \mathbf{H}(\gamma)\right]\right)>0
$$

To the second end, a K-channel filter bank formalism allows, in a similar manner, to obtain properties in $\ell^{2}(G)$ of the sequences $\left\{T_{n} \mathrm{f}_{k}\right\}_{n \in N ; k=1,2, \ldots, K}$ and $\left\{T_{n} \mathrm{~g}_{k}\right\}_{n \in N ; k=1,2, \ldots, K}$. In case they are Bessel sequences for $\ell^{2}(G)$, the idea is to consider a $K$-channel filter bank (3) where the analysis filters are $\mathrm{h}_{k}:=\widetilde{\mathrm{f}}_{k}$ and the synthesis filters are $\mathrm{g}_{k}, k=1,2, \ldots, K$. As a consequence, the corresponding polyphase matrices $\mathbf{H}(\gamma)$ and $\mathbf{G}(\gamma)$, given in Equations (5) and (6), are

$$
\mathbf{H}(\gamma)=\left(\overline{\widehat{\mathrm{f}}_{k, h_{i}}(\gamma)}\right)_{\substack{k=1,2, \ldots, K \\ i=1,2, \ldots, L}} \text { and } \mathbf{G}(\gamma)=\left(\widehat{\mathrm{g}}_{h_{i}, k}(\gamma)\right)_{\substack{i=1,2, \ldots, L \\ k=1,2, \ldots, K}} \quad \gamma \in \widehat{N}
$$

Theorem 3. Let $\left\{T_{n} \mathrm{f}_{k}\right\}_{n \in N ; k=1,2, \ldots, K}$ and $\left\{T_{n} \mathrm{~g}_{k}\right\}_{n \in N ; k=1,2, \ldots, K}$ be two Bessel sequences for $\ell^{2}(G)$, and $\mathbf{H}(\gamma)$ and $\mathbf{G}(\gamma)$ their associated matrices (11). Under the above circumstances, we have:

(a) The sequences $\left\{T_{n} f_{k}\right\}_{n \in N ; k=1,2, \ldots, K}$ and $\left\{T_{n} g_{k}\right\}_{n \in N ; k=1,2, \ldots, K}$ are dual frames for $\ell^{2}(G)$ if and only if condition $\mathbf{G}(\gamma) \mathbf{H}(\gamma)=\mathbf{I}_{L}$ a.e. $\gamma \in \widehat{N}$ holds.

(b) The sequences $\left\{T_{n} \mathrm{f}_{k}\right\}_{n \in N ; k=1,2, \ldots, K}$ and $\left\{T_{n} \mathrm{~g}_{k}\right\}_{n \in N ; k=1,2, \ldots, K}$ are biorthogonal sequences in $\ell^{2}(G)$ if and only if condition $\mathbf{H}(\gamma) \mathbf{G}(\gamma)=\mathbf{I}_{K}$ a.e. $\gamma \in \widehat{N}$ holds.

(c) The sequences $\left\{T_{n} \mathrm{f}_{k}\right\}_{n \in N ; k=1,2, \ldots, K}$ and $\left\{T_{n} \mathrm{~g}_{k}\right\}_{n \in N ; k=1,2, \ldots, K}$ are dual Riesz bases for $\ell^{2}(G)$ if and only if $K=L$ and $\mathbf{G}(\gamma)=\mathbf{H}(\gamma)^{-1}$ a.e. $\gamma \in \widehat{N}$.

(d) The sequence $\left\{T_{n} f_{k}\right\}_{n \in N ; k=1,2, \ldots, K}$ is an A-tight frame for $\ell^{2}(G)$ if and only if condition $\mathbf{H}^{*}(\gamma) \mathbf{H}(\gamma)=$ $A \mathbf{I}_{L}$ a.e. $\gamma \in \widehat{N}$ holds.

(e) The sequence $\left\{T_{n} f_{k}\right\}_{n \in N ; k=1,2, \ldots, K}$ is an orthonormal basis for $\ell^{2}(G)$ if and only if $K=L$ and $\mathbf{H}^{*}(\gamma)=$ $\mathbf{H}(\gamma)^{-1}$ a.e. $\gamma \in \widehat{N}$.

Proof. Having in mind Equation (9) and Corollary 1, part (a) is nothing but Theorem 1. 
The output of the analysis filter bank (3) corresponding to the input $g_{k^{\prime}}$ is a $K$-vector whose $k$-entry is

$$
c_{k, k^{\prime}}(m)=\downarrow_{H}\left(\mathrm{~g}_{k^{\prime}} * \mathrm{~h}_{k}\right)(m)=\left\langle\mathrm{g}_{k^{\prime}}, \mathrm{T}_{m} \widetilde{\mathrm{h}}_{k}\right\rangle_{\ell^{2}(G)}=\left\langle\mathrm{g}_{k^{\prime}}, \mathrm{T}_{m} \mathrm{f}_{k}\right\rangle_{\ell^{2}(G)},
$$

and whose $N$-Fourier transform is $\mathbf{C}_{k^{\prime}}(\gamma)=\mathbf{H}(\gamma) \mathbf{G}_{k^{\prime}}(\gamma)$ a.e. $\gamma \in \widehat{N}$, where $\mathbf{G}_{k^{\prime}}$ is the $k^{\prime}$-column of the matrix $\mathbf{G}(\gamma)$. Note that $\left\{T_{n} \mathrm{f}_{k}\right\}_{n \in N ; k=1,2, \ldots, K}$ and $\left\{T_{n} \mathrm{~g}_{k}\right\}_{n \in N ; k=1,2, \ldots, K}$ are biorthogonal if and only if $\left\langle\mathrm{g}_{k^{\prime}}, \mathrm{T}_{m} \mathrm{f}_{k}\right\rangle_{\ell^{2}(G)}=\delta\left(k-k^{\prime}\right) \delta(m)$. Therefore, the sequences $\left\{T_{n} \mathrm{f}_{k}\right\}_{n \in N ; k=1,2, \ldots, K}$ and $\left\{T_{n} \mathrm{~g}_{k}\right\}_{n \in N ; k=1,2, \ldots, K}$ are biorthogonal if and only if $\mathbf{H}(\gamma) \mathbf{G}(\gamma)=\mathbf{I}_{K}$. Thus, we have proved $(b)$.

Having in mind ([15], Theorem 7.1.1), from $(a)$ and $(b)$, we obtain $(c)$.

We can read the frame operator corresponding to the sequence $\left\{T_{n} f_{k}\right\}_{n \in N ; k=1,2, \ldots, K}$, i.e.,

$$
\mathcal{S}(\boldsymbol{\alpha})=\sum_{k=1}^{K} \sum_{n \in N}\left\langle\boldsymbol{\alpha}, T_{n} \mathrm{f}_{k}\right\rangle_{\ell^{2}(G)} T_{n} \mathrm{f}_{k}, \quad \boldsymbol{\alpha} \in \ell^{2}(G),
$$

as the output of the filter bank (3), whenever $\mathrm{h}_{k}=\widetilde{\mathrm{f}}_{k}$ and $\mathrm{g}_{k}=\mathrm{f}_{k}$, for the input $\alpha$. For this filter bank, the $\left(k, h_{l}\right)$-entry of the analysis polyphase matrix $\mathbf{H}(\gamma)$ is $\overline{\widehat{f}_{k, h_{l}}(\gamma)}$ and the $\left(h_{l}, k\right)$-entry of the synthesis polyphase matrix $\mathbf{G}(\gamma)$ is $\widehat{\mathfrak{f}}_{k, h_{l}}(\gamma)$; in other words, $\mathbf{G}(\gamma)=\mathbf{H}^{*}(\gamma)$. Hence, the sequence $\left\{T_{n} f_{k}\right\}_{n \in N ; k=1,2, \ldots, K}$ is an $A$-tight frame for $\ell^{2}(G)$, i.e.,

$$
\boldsymbol{\alpha}=\frac{1}{A} \sum_{k=1}^{K} \sum_{n \in N}\left\langle\boldsymbol{\alpha}, T_{n} \mathrm{f}_{k}\right\rangle_{\ell^{2}(G)} T_{n} \mathrm{f}_{k}, \quad \boldsymbol{\alpha} \in \ell^{2}(G)
$$

if and only if $\mathbf{H}^{*}(\gamma) \mathbf{H}(\gamma)=A \mathbf{I}_{L}$ for all $\gamma \in \widehat{N}$. Thus, we have proved $(d)$.

Finally, from $(c)$ and $(d)$, the sequence $\left\{T_{n} \mathrm{f}_{k}\right\}_{n \in N ; k=1,2, \ldots, K}$ is an orthonormal system if and only if $\mathbf{H}^{*}(\gamma)=\mathbf{H}(\gamma)^{-1}$ a.e. $\gamma \in \widehat{N}$.

\section{Getting on with Sampling}

Suppose that $\{U(n, h)\}_{(n, h) \in G}$ is a unitary representation of the group $G=N \rtimes_{\phi} H$ on a separable Hilbert space $\mathcal{H}$, and assume that for a fixed $a \in \mathcal{H}$ the sequence $\{U(n, h) a\}_{(n, h) \in G}$ is a Riesz sequence for $\mathcal{H}$ (see Ref. ([1], Theorem A)). Thus, we consider the $U$-invariant subspace in $\mathcal{H}$

$$
\mathcal{A}_{a}=\left\{\sum_{(n, h) \in G} \alpha(n, h) U(n, h) a:\{\alpha(n, h)\}_{(n, h) \in G} \in \ell^{2}(G)\right\} .
$$

For $K$ fixed elements $b_{k} \in \mathcal{H}, k=1,2, \ldots, K$, not necessarily in $\mathcal{A}_{a}$, we consider for each $x \in \mathcal{A}_{a}$ its generalized samples defined as

$$
\mathcal{L}_{k} x(m):=\left\langle x, U\left(m, 1_{H}\right) b_{k}\right\rangle_{\mathcal{H}^{\prime}} \quad m \in N \text { and } k=1,2, \ldots, K .
$$

The task is the stable recovery of any $x \in \mathcal{A}_{a}$ from the data $\left\{\mathcal{L}_{k} x(m)\right\}_{m \in N ; k=1,2, \ldots, K}$.

In what follows, we propose a solution involving a perfect reconstruction $K$-channel filter bank. First, we express the samples in a more suitable manner. Namely, for each $x=\sum_{(n, h) \in G} \alpha(n, h) U(n, h) a$ in $\mathcal{A}_{a}$, we have

$$
\begin{aligned}
\mathcal{L}_{k} x(m) & =\sum_{(n, h) \in G} \alpha(n, h)\left\langle U(n, h) a, U\left(m, 1_{H}\right) b_{k}\right\rangle \\
& =\sum_{(n, h) \in G} \alpha(n, h)\left\langle a, U\left[(n, h)^{-1} \cdot\left(m, 1_{H}\right)\right] b_{k}\right\rangle=\downarrow_{H}\left(\boldsymbol{\alpha} * \mathrm{~h}_{k}\right)(m), \quad m \in N,
\end{aligned}
$$

where $\alpha=\{\alpha(n, h)\}_{(n, h) \in G} \in \ell^{2}(G)$, and $h_{k}(n, h):=\left\langle a, U(n, h) b_{k}\right\rangle_{\mathcal{H}}$ also belongs to $\ell^{2}(G)$ for each $k=1,2, \ldots, K$. 
Suppose also that there exists a perfect reconstruction $K$-channel filter-bank with analysis filters the above $h_{k}$ and synthesis filters $g_{k}, k=1,2, \ldots, K$, such that the sequences $\left\{T_{n} \widetilde{h}_{k}\right\}_{n \in N ; k=1,2, \ldots K}$ and $\left\{T_{n} g_{k}\right\}_{n \in N ; k=1,2, \ldots K}$ are Bessel sequences for $\ell^{2}(G)$. Having in mind Equation (9), for each $\boldsymbol{\alpha}=\{\alpha(n, h)\}_{(n, h) \in G}$ in $\ell^{2}(G)$, we have

$$
\boldsymbol{\alpha}=\sum_{k=1}^{K} \sum_{n \in N} \downarrow_{H}\left(\boldsymbol{\alpha} * \mathrm{~h}_{k}\right)(n) T_{n} \mathrm{~g}_{k}=\sum_{k=1}^{K} \sum_{n \in N} \mathcal{L}_{k} x(n) T_{n} \mathrm{~g}_{k} \quad \text { in } \ell^{2}(G) .
$$

In order to derive a sampling formula in $\mathcal{A}_{a}$, we consider the natural isomorphism $\mathcal{T}_{U, a}$ : $\ell^{2}(G) \rightarrow \mathcal{A}_{a}$ which maps the usual orthonormal basis $\left\{\delta_{(n, h)}\right\}_{(n, h) \in G}$ for $\ell^{2}(G)$ onto the Riesz basis $\{U(n, h) a\}_{(n, h) \in G}$ for $\mathcal{A}_{a}$, i.e.,

$$
\mathcal{T}_{U, a}: \delta_{(n, h)} \longmapsto U(n, h) a \text { for each }(n, h) \in G .
$$

This isomorphism $\mathcal{T}_{U, a}$ possesses the following shifting property:

Lemma 2. For each $m \in N$, consider the translation operator $T_{m}$ operator defined in Equation (8). For each $m \in N$, the following shifting property holds

$$
\mathcal{T}_{U, a}\left(T_{m} \mathrm{f}\right)=U\left(m, 1_{H}\right)\left(\mathcal{T}_{U, a} \mathrm{f}\right), \quad \mathrm{f} \in \ell^{2}(G) .
$$

Proof. For each $\delta_{(n, h)}$, it is easy to check that $T_{m} \delta_{(n, h)}=\delta_{(m+n, h)}$. Hence,

$$
\mathcal{T}_{U, a}\left(T_{m} \boldsymbol{\delta}_{(n, h)}\right)=U(m+n, h) a=U\left(m, 1_{H}\right) U(n, h) a=U\left(m, 1_{H}\right)\left(\mathcal{T}_{U, a} \boldsymbol{\delta}_{(n, h)}\right) .
$$

A continuity argument proves the result for all $\mathrm{f}$ in $\ell^{2}(G)$.

Now, for each $x=\mathcal{T}_{U, a} \alpha \in \mathcal{A}_{a}$, applying the isomorphism $\mathcal{T}_{U, a}$ and the shifting property (14) in Equation (13), we get for each $x \in \mathcal{A}_{a}$ the expansion

$$
\begin{aligned}
x & =\sum_{k=1}^{K} \sum_{n \in N} \mathcal{L}_{k} x(n) \mathcal{T}_{U, a}\left(T_{n} \mathrm{~g}_{k}\right)=\sum_{k=1}^{K} \sum_{n \in N} \mathcal{L}_{k} x(n) U\left(n, 1_{H}\right)\left(\mathcal{T}_{U, a} \mathrm{~g}_{k}\right) \\
& =\sum_{k=1}^{K} \sum_{n \in N} \mathcal{L}_{k} x(n) U\left(n, 1_{H}\right) c_{k, \mathrm{~g}} \quad \text { in } \mathcal{H},
\end{aligned}
$$

where $c_{k, \mathrm{~g}}=\mathcal{T}_{U, a} \mathrm{~g}_{k}, k=1,2, \ldots, K$. In fact, the following sampling theorem in the subspace $\mathcal{A}_{a}$ holds:

Theorem 4. For $K$ fixed $b_{k} \in \mathcal{H}$, let $\mathcal{L}_{k}: \mathcal{A}_{a} \rightarrow \mathbb{C}^{N}$ be its associated $U$-system defined in Equation (12) with corresponding $\mathrm{h}_{k} \in \ell^{2}(G), k=1,2, \ldots, K$. Assume that its polyphase matrix $\mathbf{H}(\gamma)$ given in Equation (5) has all its entries in $L^{\infty}(\widehat{N})$. The following statements are equivalent:

1. The constant $A_{\mathbf{H}}=\underset{\gamma \in \widehat{N}}{\operatorname{essinf}} \lambda_{\min }\left[\mathbf{H}^{*}(\gamma) \mathbf{H}(\gamma)\right]>0$.

2. There exist $\mathrm{g}_{k}$ in $\ell^{2}(G), k=1,2, \ldots, K$, such that the associated polyphase matrix $\mathbf{G}(\gamma)$ given in (6) has all its entries in $L^{\infty}(\widehat{N})$, and it satisfies $\mathbf{G}(\gamma) \mathbf{H}(\gamma)=\mathbf{I}_{L}$ a.e. $\gamma \in \widehat{N}$.

3. There exist K elements $c_{k} \in \mathcal{A}_{a}$ such that the sequence $\left\{U\left(n, 1_{H}\right) c_{k}\right\}_{n \in N ; k=1,2, \ldots, K}$ is a frame for $\mathcal{A}_{a}$ and, for each $x \in \mathcal{A}_{a}$, the sampling formula

$$
x=\sum_{k=1}^{K} \sum_{n \in N} \mathcal{L}_{k} x(n) U\left(n, 1_{H}\right) c_{k} \quad \text { in } \mathcal{H}
$$

holds. 
4. There exists a frame $\left\{C_{k, n}\right\}_{n \in N ; k=1,2, \ldots, K}$ for $\mathcal{A}_{a}$ such that for each $x \in \mathcal{A}_{a}$ the expansion

$$
x=\sum_{k=1}^{K} \sum_{n \in N} \mathcal{L}_{k} x(n) C_{k, n} \quad \text { in } \mathcal{H}
$$

holds.

Proof. (1) implies (2). The $L \times K$ Moore-Penrose pseudo-inverse $\mathbf{H}^{\dagger}(\gamma)$ of $\mathbf{H}(\gamma)$ is given by $\mathbf{H}^{\dagger}(\gamma)=\left[\mathbf{H}^{*}(\gamma) \mathbf{H}(\gamma)\right]^{-1} \mathbf{H}^{*}(\gamma)$. Its entries are essentially bounded in $\widehat{N}$ since the entries of $\mathbf{H}(\gamma)$ belong to $L^{\infty}(\widehat{N})$ and $\operatorname{det}^{-1}\left[\mathbf{H}^{*}(\gamma) \mathbf{H}(\gamma)\right]$ is essentially bounded $\widehat{N}$ since $0<A_{\mathbf{H}}$. In addition, $\mathbf{H}^{\dagger}(\gamma) \mathbf{H}(\gamma)=\mathbf{I}_{L}$ a.e. $\gamma \in \widehat{N}$. The inverse $N$-Fourier transform in $L^{2}(\widehat{N})$ of the $k$-th column of $\mathbf{H}^{\dagger}(\gamma)$ gives $g_{k}, k=1,2, \ldots, K$.

(2) implies (3). According to Theorems 2 and 3, the sequences $\left\{T_{n} \widetilde{\mathrm{h}}_{k}\right\}_{n \in N ; k=1,2 \ldots K}$ and $\left\{T_{n} \mathrm{~g}_{k}\right\}_{n \in N ; k=1,2, \ldots K}$ form a pair of dual frames for $\ell^{2}(G)$. We deduce the sampling expansion as in Formula (15). In addition, the sequence $\left\{U\left(n, 1_{H}\right) c_{k, \mathrm{~g}}\right\}_{n \in N ; k=1,2, \ldots, K}$ is a frame for $\mathcal{A}_{a}$.

Obviously, (3) implies (4). Finally, (4) implies (1). Applying $\mathcal{T}_{U, a}^{-1}$, we get that the sequences $\left\{T_{n} \widetilde{\mathrm{h}}_{k}\right\}_{n \in N ; k=1,2, \ldots K}$ and $\left\{\mathcal{T}_{U, a}^{-1}\left(C_{k, n}\right)\right\}_{n \in N ; k=1,2, \ldots, K}$ form a pair of dual frames for $\ell^{2}(G)$; in particular, by using Theorem 2 , we obtain that $0<A_{\mathbf{H}}$.

All the possible solutions of $\mathbf{G}(\gamma) \mathbf{H}(\gamma)=\mathbf{I}_{L}$ a.e. $\gamma \in \widehat{N}$ with entries in $L^{\infty}(\widehat{N})$ are given in terms of the Moore-Penrose pseudo inverse by the $L \times K$ matrices $\mathbf{G}(\gamma):=\mathbf{H}^{\dagger}(\gamma)+\mathbf{U}(\gamma)\left[\mathbf{I}_{K}-\mathbf{H}(\gamma) \mathbf{H}^{\dagger}(\gamma)\right]$, where $\mathbf{U}(\gamma)$ denotes any $L \times K$ matrix with entries in $L^{\infty}(\widehat{N})$.

Notice that $K \geq L$ where $L$ is the order of the group $H$. In case $K=L$, we obtain:

Corollary 2. In the case $K=L$, assume that its polyphase matrix $\mathbf{H}(\gamma)$ given in Equation (5) has all entries in $L^{\infty}(\widehat{N})$. The following statements are equivalent:

1. The constant $A_{\mathbf{H}}=\underset{\gamma \in \widehat{N}}{\operatorname{essinf}} \lambda_{\min }\left[\mathbf{H}^{*}(\gamma) \mathbf{H}(\gamma)\right]>0$.

2. There exist $L$ unique elements $c_{k}, k=1,2, \ldots, L$, in $\mathcal{A}_{a}$ such that the associated sequence $\left\{U\left(n, 1_{H}\right) c_{k}\right\}_{n \in N ; k=1,2, \ldots, L}$ is a Riesz basis for $\mathcal{A}_{a}$ and the sampling formula

$$
x=\sum_{k=1}^{L} \sum_{n \in N} \mathcal{L}_{k} x(n) U\left(n, 1_{H}\right) c_{k} \quad \text { in } \mathcal{H}
$$

holds for each $x \in \mathcal{A}_{a}$.

Moreover, the interpolation property $\mathcal{L}_{k} c_{k^{\prime}}(n)=\delta_{k, k^{\prime}} \delta_{n, 0_{N}}$, where $n \in N$ and $k, k^{\prime}=1,2, \ldots, L$, holds.

Proof. In this case, the square matrix $\mathbf{H}(\gamma)$ is invertible and the result comes out from Theorem 3. From the uniqueness of the coefficients in a Riesz basis expansion, we get the interpolation property.

Denote $H=\left\{h_{1}, h_{2}, \ldots, h_{L}\right\}$; for a fixed $b \in \mathcal{H}$, we consider the samples

$$
\mathcal{L}_{k} x(m):=\left\langle x, U\left(m, h_{k}\right) b\right\rangle, \quad m \in N \text { and } k=1,2, \ldots, L,
$$

of any $x \in \mathcal{A}_{a}$. Since $U\left(m, h_{k}\right) b=U\left(m, 1_{H}\right) U\left(0_{N}, h_{k}\right) b=U\left(m, 1_{H}\right) b_{k}$, where $b_{k}:=U\left(0_{N}, h_{k}\right) b$, $k=1,2, \ldots, L$, we are in a particular case of Equation (12) with $K=L$.

Notice also that the subspace $\mathcal{A}_{a}$ can be viewed as the multiple generated $U$-invariant subspace of $\mathcal{H}$

$$
\overline{\operatorname{span}}\left\{U\left(n, 1_{H}\right) a_{h}: n \in N, h \in H\right\}
$$

with $L$ generators $a_{h}:=U\left(0_{N}, h\right) a \in \mathcal{H}, h \in H$, obtained from $a \in \mathcal{H}$ by the action of the group $H$. 


\subsection{An Example Involving Crystallographic Groups}

The Euclidean motion group $E(d)$ is the semi-direct product $\mathbb{R}^{d} \rtimes_{\phi} O(d)$ corresponding to the homomorphism $\phi: O(d) \rightarrow A u t\left(\mathbb{R}^{d}\right)$ given by $\phi_{A}(x)=A x$, where $A \in O(d)$ and $x \in \mathbb{R}^{d}$. The composition law on $E(d)=\mathbb{R}^{d} \rtimes_{\phi} O(d)$ reads $(x, A) \cdot\left(x^{\prime}, A^{\prime}\right)=\left(x+A x^{\prime}, A A^{\prime}\right)$.

Let $M$ be a non-singular $d \times d$ matrix and $\Gamma$ a finite subgroup of $O(d)$ of order $L$ such that $A\left(M \mathbb{Z}^{d}\right)=M \mathbb{Z}^{d}$ for each $A \in \Gamma$. We consider the crystallographic group $\mathcal{C}_{M, \Gamma}:=M \mathbb{Z}^{d} \rtimes_{\phi} \Gamma$ and its quasi regular representation (see Ref. [1]) on $L^{2}\left(\mathbb{R}^{d}\right)$

$$
U(n, A) f(t)=f\left[A^{\top}(t-n)\right], \quad n \in M \mathbb{Z}^{d}, A \in \Gamma \text { and } f \in L^{2}\left(\mathbb{R}^{d}\right) .
$$

For a fixed $\varphi \in L^{2}\left(\mathbb{R}^{d}\right)$ such that the sequence $\{U(n, A) \varphi\}_{(n, A) \in \mathcal{C}_{M, \Gamma}}$ is a Riesz sequence for $L^{2}\left(\mathbb{R}^{d}\right)$ (see, for instance, Refs. $\left.[19,20]\right)$ we consider the $U$-invariant subspace in $L^{2}\left(\mathbb{R}^{d}\right)$

$$
\begin{aligned}
\mathcal{A}_{\varphi} & =\left\{\sum_{(n, A) \in \mathcal{C}_{M, \Gamma}} \alpha(n, A) \varphi\left[A^{\top}(t-n)\right]:\{\alpha(n, A)\} \in \ell^{2}\left(\mathcal{C}_{M, \Gamma}\right)\right\} \\
& =\left\{\sum_{(n, A) \in \mathcal{C}_{M, \Gamma}} \alpha(n, A) \varphi(A t-n):\{\alpha(n, A)\} \in \ell^{2}\left(\mathcal{C}_{M, \Gamma}\right)\right\} .
\end{aligned}
$$

Choosing $K$ functions $b_{k} \in L^{2}\left(\mathbb{R}^{d}\right), k=1,2, \ldots, K$, we consider the average samples of $f \in \mathcal{A}_{\varphi}$

$$
\mathcal{L}_{k} f(n)=\left\langle f, U(n, I) b_{k}\right\rangle=\left\langle f, b_{k}(\cdot-n)\right\rangle, \quad n \in M \mathbb{Z}^{d} .
$$

Under the hypotheses in Theorem 4, there exist $K \geq L$ sampling functions $\psi_{k} \in \mathcal{A}_{\varphi}$ for $k=1,2, \ldots, K$, such that the sequence $\left\{\psi_{k}(\cdot-n)\right\}_{n \in M \mathbb{Z}^{d} ; k=1,2, \ldots, K}$ is a frame for $\mathcal{A}_{\varphi}$, and the sampling expansion

$$
f(t)=\sum_{k=1}^{K} \sum_{n \in M \mathbb{Z}^{d}}\left\langle f, b_{k}(\cdot-n)\right\rangle_{L^{2}\left(\mathbb{R}^{d}\right)} \psi_{k}(t-n) \quad \text { in } L^{2}\left(\mathbb{R}^{d}\right)
$$

holds.

If the generator $\varphi \in C\left(\mathbb{R}^{d}\right)$ and the function $t \mapsto \sum_{n}|\varphi(t-n)|^{2}$ is bounded on $\mathbb{R}^{d}$, a standard argument shows that $\mathcal{A}_{\varphi}$ is a reproducing kernel Hilbert space (RKHS) of bounded continuous functions in $L^{2}\left(\mathbb{R}^{d}\right)$. As a consequence, convergence in $L^{2}\left(\mathbb{R}^{d}\right)$-norm implies pointwise convergence which is absolute and uniform on $\mathbb{R}^{d}$.

Notice that the infinite dihedral group $D_{\infty}=\mathbb{Z} \rtimes_{\phi} \mathbb{Z}_{2}$ is a particular crystallographic group with lattice $\mathbb{Z}$ and $\Gamma=\mathbb{Z}_{2}$. Its quasi regular representation on $L^{2}(\mathbb{R})$ reads

$$
U(n, 0) f(t)=f(t-n) \quad \text { and } \quad U(n, 1) f(t)=f(-t+n), \quad n \in \mathbb{Z} \text { and } f \in L^{2}(\mathbb{R}) .
$$

Thus, we could obtain sampling formulas as (17) for $K \geq 2$ average functions $b_{k}$.

The quasi regular unitary representation of a crystallographic group $\mathcal{C}_{M, \Gamma}$ on $L^{2}\left(\mathbb{R}^{d}\right)$ motivates the next section:

\subsection{The Case of Pointwise Samples}

Let $\{U(n, h)\}_{(n, h) \in G}$ be a unitary representation of the group $G=N \rtimes_{\phi} H$ on the Hilbert space $\mathcal{H}=L^{2}\left(\mathbb{R}^{d}\right)$. If the generator $\varphi \in L^{2}\left(\mathbb{R}^{d}\right)$ satisfies that, for each $(n, h) \in G$, the function $U(n, h) \varphi$ is continuous on $\mathbb{R}^{d}$, and the condition

$$
\sup _{t \in \mathbb{R}^{d}} \sum_{(n, h) \in G}|[U(n, h) \varphi](t)|^{2}<\infty,
$$


then the subspace $\mathcal{A}_{\varphi}$ is an RKHS of bounded continuous functions in $L^{2}\left(\mathbb{R}^{d}\right)$; proceeding as in [21], one can prove that the above conditions are also necessary.

For $K$ fixed points $t_{k} \in \mathbb{R}^{d}, k=1,2, \ldots, K$, we consider for each $f \in \mathcal{A}_{\varphi}$ the new samples given by

$$
\mathcal{L}_{k} f(n):=\left[U\left(-n, 1_{H}\right) f\right]\left(t_{k}\right), \quad n \in N \text { and } k=1,2, \cdots, K .
$$

For each $f=\sum_{(m, h) \in G} \alpha(m, h) U(m, h) \varphi$ in $\mathcal{A}_{\varphi}$ and $k=1,2, \ldots, K$, we have

$$
\begin{aligned}
\mathcal{L}_{k} f(n) & =\left[\sum_{(m, h) \in G} \alpha(m, h) U\left[\left(-n, 1_{H}\right) \cdot(m, h)\right] \varphi\right]\left(t_{k}\right) \\
& =\sum_{(m, h) \in G} \alpha(m, h)[U(m-n, h) \varphi]\left(t_{k}\right)=\left\langle\boldsymbol{\alpha}, T_{n} \mathrm{f}_{k}\right\rangle_{\ell^{2}(G)}, \quad n \in N,
\end{aligned}
$$

where $\boldsymbol{\alpha}=\{\alpha(m, h)\}_{(m, h) \in G}$ and $\mathrm{f}_{k}(m, h):=\overline{[U(m, h) \varphi]\left(t_{k}\right)},(m, h) \in G$. Notice that $\mathrm{f}_{k}$ belongs to $\ell^{2}(G), k=1,2, \cdots, K$. As a consequence, under the hypotheses in Theorem 4 (on these new $\mathrm{h}_{k}:=\widetilde{\mathrm{f}}_{k}$, $k=1,2, \ldots, K)$, a sampling formula such as (16) holds for the data sequence $\left\{\mathcal{L}_{k} f(n)\right\}_{n \in N ; k=1,2, \ldots, K}$ defined in Equation (18).

In the particular case of the quasi regular representation of a crystallographic group $\mathcal{C}_{M, \Gamma}=M \mathbb{Z}^{d} \rtimes_{\phi} \Gamma$, for each $f \in \mathcal{A}_{\varphi}$, the samples (18) read

$$
\mathcal{L}_{k} f(n)=[U(-n, I) f]\left(t_{k}\right)=f\left(t_{k}+n\right), \quad n \in M \mathbb{Z}^{d} \text { and } k=1,2, \ldots, K .
$$

Thus (under hypotheses in Theorem 4), there exist $K$ functions $\psi_{k} \in \mathcal{A}_{\varphi}, k=1,2, \ldots, K$, such that for each $f \in \mathcal{A}_{\varphi}$ the sampling formula

$$
f(t)=\sum_{k=1}^{K} \sum_{n \in M \mathbb{Z}^{d}} f\left(t_{k}+n\right) \psi_{k}(t-n), \quad t \in \mathbb{R}^{d}
$$

holds. The convergence of the series in the $L^{2}\left(\mathbb{R}^{d}\right)$-norm sense implies pointwise convergence which is absolute and uniform on $\mathbb{R}^{d}$.

\section{Conclusions}

In this paper, we have derived an abstract regular sampling theory associated with a unitary representation $(n, h) \mapsto U(n, h)$ of a group $G$ which is a semi-direct product of two groups, $N$ countable discrete abelian group and $H$ finite, on a separable Hilbert space $\mathcal{H}$; here, regular sampling means that we are taken the samples at the group $N$. Concretely, the sampling theory is obtained in the $U$-invariant subspace of $\mathcal{H}$ generated by $a \in \mathcal{H}$ that is

$$
\mathcal{A}_{a}=\left\{\sum_{(n, h) \in G} \alpha(n, h) U(n, h) a:\{\alpha(n, h)\}_{(n, h) \in G} \in \ell^{2}(G)\right\}
$$

and the samples of $x \in \mathcal{A}_{a}$ are given by $\mathcal{L}_{k} x(n):=\left\langle x, U\left(n, 1_{H}\right) b_{k}\right\rangle_{\mathcal{H}^{\prime}}, n \in N$, where $b_{k}, k=1,2, \ldots, K$, denote $K$ fixed elements in $\mathcal{H}$ which do not belong necessarily to $\mathcal{A}_{a}$. We look for $K$ elements $c_{k} \in \mathcal{A}_{a}$ such that the sequence $\left\{U\left(n, 1_{H}\right) c_{k}\right\}_{n \in N ; k=1,2, \ldots, K}$ is a frame for $\mathcal{A}_{a}$ and, for each $x \in \mathcal{A}_{a}$, the sampling formula $x=\sum_{k=1}^{K} \sum_{n \in N} \mathcal{L}_{k} x(n) U\left(n, 1_{H}\right) c_{k}$ holds.

A similar problem was solved when the group $G$ is a discrete LCA group and the samples are taken at a uniform lattice of $G$ (see Ref. [10]). In the case of an abelian group, we have the Fourier transform, a basic tool in this previous work. In the present work, a classical Fourier analysis on $G$ is not available, but if $G$ is a semi-direct product of the form $G=N \rtimes_{\phi} H$, where $N$ is a countable discrete abelian group and $H$ is a finite group, the Fourier transform on the abelian group $N$ allows us to solve the problem by means of a filter bank formalism. Recalling the filter bank formalism in discrete LCA 
groups, the defined samples are expressed as the output of a suitable $K$-channel analysis filter bank corresponding to the input $x \in \mathcal{A}_{a}$. The frame analysis of this filter bank along with the synthesis one giving perfect reconstruction allows us to obtain a pair of suitable dual frames for obtaining the desired sampling result, which is written as a list of equivalent statements (see Theorem 4).

Although the semi-direct product of groups represents, so to speak, the simplest case of non-abelian groups, this paper can be a good starting point for finding sampling theorems associated with unitary representations of non abelian groups that are not isomorphic to a semi-direct product of groups.

Author Contributions: The authors contributed equally in the aspects concerning this work: conceptualization, methodology, writing — original draft preparation, writing — review and editing and funding acquisition.

Funding: This research was funded by the grant MTM2017-84098-P from the Spanish Ministerio de Economía y Competitividad (MINECO).

Acknowledgments: The authors wish to thank the referees for their valuable and constructive comments.

Conflicts of Interest: The authors declare no conflict of interest.

\section{References}

1. Barbieri, D.; Hernández, E.; Parcet, J. Riesz and frame systems generated by unitary actions of discrete groups. Appl. Comput. Harmon. Anal. 2015, 39, 369-399. [CrossRef]

2. Aldroubi, A.; Sun, Q.; Tang, W.S. Convolution, average sampling, and a Calderon resolution of the identity for shift-invariant spaces. J. Fourier Anal. Appl. 2005, 11, 215-244. [CrossRef]

3. Fernández-Morales, H.R.; García, A.G.; Hernández-Medina, M.A.; Muñoz-Bouzo, M.J. Generalized sampling: From shift-invariant to $U$-invariant spaces. Anal. Appl. 2015, 13, 303-329. [CrossRef]

4. García, A.G.; Pérez-Villalón, G. Dual frames in $L^{2}(0,1)$ connected with generalized sampling in shift-invariant spaces. Appl. Comput. Harmon. Anal. 2006, 20, 422-433. [CrossRef]

5. García, A.G.; Pérez-Villalón, G. Multivariate generalized sampling in shift-invariant spaces and its approximation properties. J. Math. Anal. Appl. 2009, 355, 397-413. [CrossRef]

6. Kang, S.; Kwon, K.H. Generalized average sampling in shift-invariant spaces. J. Math. Anal. Appl. 2011, 377, 70-78. [CrossRef]

7. Michaeli, T.; Pohl, V.; Eldar Y.C. U-invariant sampling: Extrapolation and causal interpolation from generalized samples. IEEE Trans. Signal Process. 2011, 59, 2085-2100. [CrossRef]

8. Pohl, V.; Boche, H. U-invariant sampling and reconstruction in atomic spaces with multiple generators. IEEE Trans. Signal Process. 2012, 60, 3506-3519. [CrossRef]

9. Sun, W.; Zhou, X. Average sampling in shift-invariant subspaces with symmetric averaging functions. J. Math. Anal. Appl. 2003, 287, 279-295. [CrossRef]

10. García, A.G.; Hernández-Medina, M.A.; Pérez-Villalón, G. Sampling in unitary invariant subspaces associated with LCA groups. Results Math. 2017, 72, 1725-1745. [CrossRef]

11. Faridani, A. A generalized sampling theorem for locally compact abelian groups. Math. Comp. 1994, 63, 307-327. [CrossRef]

12. Bölcskei, H.; Hlawatsch, F.; Feichtinger, H.G. Frame-theoretic analysis of oversampled filter banks. IEEE Trans. Signal Process. 1998, 46, 3256-3268. [CrossRef]

13. Cvetković, Z.; Vetterli, M. Oversampled filter banks. IEEE Trans. Signal Process. 1998, 46, 1245-1255. [CrossRef]

14. García, A.G.; Hernández-Medina, M.A.; Pérez-Villalón, G. Filter Banks on Discrete Abelian Groups. Internat. J. Wavelets Multiresolut. Inf. Process. 2018, 16, 1850029. [CrossRef]

15. Christensen, O. An Introduction to Frames and Riesz Bases, 2nd ed.; Birkhäuser: Boston, MA, USA, 2016.

16. Folland, G.B. A Course in Abstract Harmonic Analysis; CRC Press: Boca Raton, FL, USA, 1995.

17. Führ, H. Abstract Harmonic Analysis of Continuous Wavelet Transforms; Springer: Berlin, Germany, 2005.

18. Horn, R.A.; Johnson, C.R. Matrix Analysis; Cambridge University Press: Cambridge, UK, 1999.

19. De Boor, C.; DeVore, R.A.; Ron A. On the construction of multivariate pre-wavelets. Constr. Approx. 1993, 9, 123-166. [CrossRef] 
20. Jia, R.Q.; Micchelli, C.A. Using the refinement equations for the construction of pre-waveles II: Powers of two. In Curves and Surfaces; Laurent, P.J., Le Méhauté, L., Schumaker, L., Eds.; Academic Press: Boston, MA, USA, 1991; pp. 209-246.

21. Zhou, X.; Sun, W. On the sampling theoren for wavelet subspaces. J. Fourier Anal. Appl. 1999, 5, $347-354$. [CrossRef]

(C) 2019 by the authors. Licensee MDPI, Basel, Switzerland. This article is an open access article distributed under the terms and conditions of the Creative Commons Attribution (CC BY) license (http:/ / creativecommons.org/licenses/by/4.0/). 